\title{
TOTAL REGULARITY OF GENERAL TRANSFORMATIONS ${ }^{1}$
}

\author{
HENRY HURWITZ, JR.
}

A method of summation is said to be regular if it assigns to every convergent series its actual value. If it also assigns the value $+\infty$ (or $-\infty$ ) to every series which diverges to $+\infty$ (or $-\infty)$ it is said to be totally regular. The conditions for regularity are well known, and those for total regularity have been worked out for triangular matrix transformations by W. A. Hurwitz. ${ }^{2}$ We here obtain necessary and sufficient conditions for total regularity for a more general type of transformation. (The former conditions, though still sufficient, are not necessary.)

Suppose $x_{1}, x_{2}, x_{3}, \ldots$ is the sequence of partial sums of the original series which is assumed real. A value $Y$ is assigned to this sequence in the following way:

$$
Y=\lim _{D(t) \rightarrow 0} y(t) ; \quad y(t)=\sum_{k=1}^{\infty} a_{k}(t) x_{k} .
$$

$t$ is a variable ranging over some point set, $D(t)$ is a positive real function, and the functions $a_{k}(t)$ are real, but not necessarily continuous. We assume the transformation is regular so that the three Silverman-Toeplitz conditions are satisfied:

(1) $\sum_{k=1}^{\infty}\left|a_{k}(t)\right|$ is bounded for $D(t)$ sufficiently small;

(2) $\lim _{D(t) \rightarrow 0} \sum_{k=1}^{\infty} a_{k}(t)=1$;

(3) $\lim _{D(t) \rightarrow 0} a_{k}(t)=0$ for all $k$.

We then ask when $Y$ will be positively infinite if $\lim _{k \rightarrow \infty} x_{k}=+\infty$. (We demand that for $D(t)$ sufficiently small $y(t)$ will be defined although it may be positively infinite.)

First it may be seen that for sufficiently advanced $t$ (that is, $t$ for which $D(t)$ is sufficiently small) there can be only a finite number of negative coefficients $a_{k}(t)$ in each row (that is, for each $t$ ) if the transformation is to be totally regular. Otherwise a sequence $t_{n}$ with $D\left(t_{n}\right) \rightarrow 0$ could be picked out such that for each $t_{n}$ there would be an infinite number of negative coefficients. Then a sequence $x_{k}$ could be defined so that $x_{k} \rightarrow \infty$ and

$$
\sum_{k=1}^{\infty} a_{k}\left(t_{n}\right) x_{k}
$$

${ }^{1}$ Presented to the Society, February 24, 1940, under the title Total regularity of infinite matrix transformations.

${ }^{2}$ W. A. Hurwitz, Proceedings of the London Mathematical Society, vol. 26 (1927), p. 231. 
is for all $t_{n}$, if defined at all, certainly less than, say, zero. This would be accomplished by defining $x_{k}$ unusually large for some $k$ for which $a_{k}\left(t_{1}\right)<0$, then for a $k$ for which $a_{k}\left(t_{2}\right)<0$, and so on in the order $t_{1}, t_{2}, t_{1}, t_{2}, t_{3}, t_{1}, t_{2}, t_{3}, t_{4}, \cdots$, each time taking a greater $k$. Imposing this necessary condition considerably limits the possibilities and assures that for any $x_{k} \rightarrow \infty, y(t)$ will be defined for $t$ sufficiently advanced.

DEFINITION. The guard of the coefficient $a_{k}\left(t_{0}\right)$ with respect to the finite number of rows $t_{1}, t_{2}, \cdots, t_{n}$, written

$$
\left.G a_{k}\left(t_{0}\right)\right]_{t_{1} t_{2} \cdots t_{n}} \text {, }
$$

is equal to +1 if $a_{k}\left(t_{0}\right) \geqq 0$. If $a_{k}\left(t_{0}\right)<0$ it is equal to the maximum value of

$$
\frac{a_{k}\left(t^{\prime}\right)}{\left|a_{l i}\left(t_{0}\right)\right|}
$$

for $t^{\prime}=t_{1}, t_{2}, \cdots, t_{n}$.

THEOREM 1. If a real regular transformation is to be totally regular, it is necessary and sufficient that

(a) for $D(t)$ sufficiently small there is only a finite number of negative coefficients in each row;

(b) there does not exist a sequence $t_{1}, t_{2}, \cdots$ such that $\lim _{n \rightarrow \infty} D\left(t_{n}\right)=0$ and for every finite number of rows $t_{1}, t_{2}, \cdots, t_{F}$

$$
\left.\limsup _{n \rightarrow \infty} G a_{k}^{*}\left(t_{n}\right)\right]_{t_{1} t_{2} \cdots t_{F}} \leqq 0
$$

for some sequence $k_{1}^{*}, k_{2}^{*}, \cdots$ such that $k_{n}{ }^{*} \rightarrow \infty$, where $k_{n}{ }^{*}$ may depend on $F$.

THEOREM 2. Theorem 1 holds with $\lim \sup _{n \rightarrow \infty} G$ replaced by $\lim \inf _{n \rightarrow \infty} G$.

The equivalence of these two theorems may be demonstrated directly, but it also follows by proving that the conditions of Theorem 1 are sufficient, and those of Theorem 2 are necessary for total regularity.

To prove the sufficiency part of Theorem 1, we show that if a transformation satisfies the conditions there cannot exist a sequence $x_{k} \rightarrow \infty$ such that there is a sequence $t_{n}$ with $D\left(t_{n}\right) \rightarrow 0$ and $y\left(t_{n}\right)<M$ for all $n$. $M$ is some large positive number. Suppose such sequences $x_{k}$ and $t_{n}$ do exist. We choose $K_{n}$ such that

$$
\sum_{k=1}^{K_{n}}\left|a_{k}\left(t_{n}\right) x_{k}\right|<M, \quad \sum_{k=1}^{K_{n}}\left|a_{k}\left(t_{n}\right)\right|<\frac{1}{3} .
$$


By the third S-T condition we may have $K_{n} \rightarrow \infty$, and indeed may assume without loss of generality that all the $K_{n}$ 's are so large that if $k$ is greater than any one of them $x_{k}>9 M$. By the second S-T condition, we may assume that

$$
\left|\sum_{k=1}^{\infty} a_{k}\left(t_{n}\right)-1\right|<\frac{1}{3}
$$

for all $n$. By hypothesis there is some integer $F$ such that there is no sequence $k_{n}^{*} \rightarrow \infty$ for which

$$
\left.\limsup _{n \rightarrow \infty} G a_{k_{n}}^{*}\left(t_{n}\right)\right]_{t_{1} t_{2} \ldots t_{F}} \leqq 0 .
$$

It then follows that at least for an infinite subsequence $t_{n_{l}}$ every coefficient $a_{k}\left(t_{n_{l}}\right)$ in the row $t_{n_{l}}$ with $k>K_{n}$ has

$$
\left.G a_{k}\left(t_{n_{l}}\right)\right]_{t_{1} t_{2} \cdots t_{F}}>\beta
$$

where $\beta$ is some positive number, unique for the whole subsequence. Since $y\left(t_{n_{1}}\right)<M$, we must have

$$
J_{1}=\sum_{K_{n_{1}}+1}^{\infty} a_{k}\left(t_{n_{1}}\right) x_{k}<-M
$$

where the asterisk indicates that the summation is to be extended over only those $k$ 's for which $a_{k}\left(t_{n_{1}}\right)<0$. Because the guard with respect to the rows $t_{1}, t_{2}, \cdots, t_{F}$ of each of the coefficients appearing in the expression for $J_{1}$ is greater than $\beta$, we have

$$
\sum_{J_{1}} a_{k}\left(t_{i_{k}}\right) x_{k}>\beta M
$$

where the summation is over all the $k$ 's appearing in the expression for $J_{1}$ and the $i_{k}$ 's are suitably chosen integers ranging between 1 and $F$. We next pick from the sequence $n_{l}$ an element $n_{2}^{\prime}$ such that $K_{n_{2}^{\prime}}$ is greater than any $k$ appearing in $J_{1}$. As before we have

so that

$$
J_{2}=\sum_{K_{n_{2}^{\prime}+1}^{\prime}}^{\infty} *_{k}\left(t_{n_{2}^{\prime}}\right) x_{k}<-M
$$

$$
\sum_{J_{1}, J_{2}} a_{k}\left(t_{i_{k}}\right) x_{k}>2 \beta M
$$

where the summation is now over all the $k$ 's appearing in either $J_{1}$ or $J_{2}$. Proceeding in this way we can prove that it is possible to pick from the rows $t_{1}, t_{2}, \cdots, t_{F}$ a series diverging to positive infinity. But 
since there is only a finite number of negative terms in each row it is then impossible that

$$
y\left(t_{i}\right)<M
$$

for $i=1, \cdots, F$, so that an $x_{k}$ sequence of the type assumed cannot exist.

To prove that the conditions of Theorem 2 are necessary, we assume that a sequence $t_{n}$ of the kind forbidden by (b) of Theorem 1 , with $\lim \sup G$ replaced by $\lim \inf G$, exists and actually construct a sequence $x_{k} \rightarrow \infty$ such that

$$
\lim _{D(t) \rightarrow 0} y(t) \neq+\infty .
$$

By the second S-T condition we may assume

$$
\sum_{k=1}^{\infty}\left|a_{k}\left(t_{n}\right)\right|<T>1
$$

for all $n$. Let $n_{1}=1$. Take $K_{1}$ such that

$$
\sum_{k=K_{1}+1}^{\infty}\left|a_{k}\left(t_{n_{1}}\right)\right|<\frac{1}{2 \cdot 2^{2}}=\frac{1}{8}
$$

Define

$$
x_{k}=\frac{1}{T}, \quad k=1,2, \cdots, K_{1}
$$

By the assumption we have in general, for $s>1$,

$$
\left.\liminf _{n \rightarrow \infty} G a_{k_{n}^{(s-1)}}\left(t_{n}\right)\right]_{t_{n_{1}} t_{n_{2}} \cdots t_{n_{s-1}}} \leqq 0
$$

for some sequence $k_{n}^{(s-1)}$ which becomes infinite with $n$. We can therefore choose $n_{s}>n_{s-1}$ so that

(a) $\left.G a_{k_{s}}^{(s-1)}\left(t_{n_{s}}\right)\right]_{t_{n_{1}} t_{n_{2}} \cdots t_{n_{s-1}}}<1 / s \cdot 2^{s}$,

(b) $k_{n_{s}}^{(s-1)}>K_{s-1}$,

(c) $D\left(t_{n_{s}}\right)$ is so small that $\sum_{k=1}^{K_{s-1}}\left|a_{k}\left(t_{n_{s}}\right) x_{k}\right|<1$.

Call $k_{n_{s}}^{(s-1)}$ simply $k_{s}$. Take $K_{s}>k_{s}$ such that

$$
\sum_{k=K}^{\infty}\left|a_{k+1}\left(t_{n p}\right)\right|<\frac{1}{(s+1) 2^{s+1}}
$$

for $p=1,2, \cdots, s$. Define in general

$$
x_{k}=s / T, \quad K_{s-1}+1 \leqq k \leqq K_{s} \quad\left(k \neq k_{s}\right), \quad x_{k_{s}}=X_{s}
$$


where $X_{s}$ is the greater of $s /\left|a_{k_{s}}\left(t_{n_{s}}\right)\right|, s / T$. It is apparent that the $x_{k}$ sequence thus defined approaches infinity. Also, since $a_{k_{s}}\left(t_{n_{s}}\right)$ is negative and has a small guard, it may be verified that $y\left(t_{n_{s}}\right)$ remains bounded for all $s$, so that the transformation is not totally regular.

THEOREM 3. Theorems 1 and 2 hold with the modification that the sequence $k_{n}^{*}$ must be independent of $F$.

To prove this it must be shown that if a sequence $t_{n}$ of the type forbidden by Theorems 1 or 2 exists, then one with $k_{n}^{*}$ independent of $F$ can be found. But this can easily be done by taking a subsequence $t_{n_{1}}, t_{n_{2}}, t_{n_{3}}, \cdots$ from the sequence $t_{1}, t_{2}, t_{3}, \cdots$ so that some negative coefficient $a_{k_{l}}\left(t_{n_{l}}\right)$ with large $k_{l}^{\prime}$ in row $t_{n_{l}}$ has a small guard with respect to all the rows $t_{n_{1}}, t_{n_{2}}, \cdots, t_{n_{l-1}}$. The $k_{l}^{\prime}$ and $t_{n_{l}}$ sequences thus obtained will be of the type forbidden by Theorem 3. The same line of reasoning also serves to prove a second modification:

THEOREM 4. Theorem 3 will hold with

$$
\left.\limsup _{n \rightarrow \infty} G a_{k_{n}^{*}}^{*}\left(t_{n}\right)\right]_{t_{1} t_{2}} \cdots t_{n-1} \leqq 0
$$

instead of

$$
\left.\limsup _{n \rightarrow \infty} G a_{k_{n}^{*}}^{*}\left(t_{n}\right)\right]_{t_{1} t_{2}} \ldots t_{F} \leqq 0 .
$$

(The same thing holds with inferior limits.)

The most powerful combination of these four theorems is obtained by using the necessary conditions of Theorem 2 and the sufficient conditions (in terms of superior limits) of Theorem 4. The theorem obtained by W. A. Hurwitz for triangular matrix transformations follows quite easily from these results.

THEOREM 5. It is necessary (but not sufficient) for a real regular transformation to be totally regular that there does not exist a sequence of coefficients $a_{k_{n}}\left(t_{n}\right), n=1,2, \cdots$, such that (a) $a_{k_{n}}\left(t_{n}\right)<0$ for all $n$; (b) the maximum number of $n$ 's for which $k_{n}$ has any particular value is finite; (c) $\lim _{n \rightarrow \infty} D\left(t_{n}\right)=0$; (d) $\sum_{n=1}^{\infty} a_{k_{n}}\left(t_{n}\right)=-\infty$.

THEOREM 6. It is necessary for a real regular transformation to be totally regular that

$$
\lim _{D(t) \rightarrow 0} \sum_{k=1}^{\infty}\left[\left|a_{k}(t)\right|-a_{k}(t)\right]=0 .
$$

HARVARD UNIVERSITY 